\title{
Resiliencia. Diferencias entre estudiantes universitarios y jóvenes trabajadores ${ }^{1}$
}

\author{
José Alonso Hernández León², Juan Francisco Caldera Montes³, Oscar Ulises Reynoso González4, \\ Iván Alejandro Caldera Zamora ${ }^{5}$, Santiago Salcedo Orozco ${ }^{6}$ \\ Centro Universitario de Los Altos de la Universidad de Guadalajara (México)
}

Recibido: 01/03/2019 Aceptado: 13/12/2019

\section{Resumen}

Objetivo. Comparar los niveles de resiliencia entre jóvenes universitarios y jóvenes trabajadores, e identificar posibles relaciones de dicha variable con la edad de los participantes. Método. La muestra, de tipo incidental, estuvo conformada por 188 jóvenes, cuya edad oscilaba entre los 18 y los 27 años. Al momento del estudio, 138 cursaban una carrera profesional universitaria y 50 eran trabajadores. Para medir la variable del estudio se utilizó la Escala de Resiliencia traducida y adaptada al español. Resultados. El principal hallazgo es que los jóvenes universitarios obtienen puntajes significativamente mayores que los trabajadores, tanto en el total de la prueba de resiliencia $(t(186)=-3.98, p=0.000, d=0.65)$ como en las dos dimensiones que la componen, Competencia personal $(t(186)=-4.45, p=0.000, d=0.73)$ y Aceptación de sí mismo y de la vida $(t(186)=$ -2.23, $p=0.026, d=0.36)$. Conclusión. Un mayor nivel de estudios podría considerarse un factor protector ante la adversidad, al relacionarse con una mayor resiliencia.

Palabras clave. Resiliencia, estudiantes universitarios, jóvenes, resistencia.

\section{Resilience. Differences between Graduate Students and Young Workers}

\footnotetext{
Abstract

Objective. To compare the levels of resilience between university and young workers, as well as between men and women, and to identify possible relationships of this variable with the age of the participants. Method. This was a correlational study with transversal-correlational design. The sample was incidental and was made up of 188 people between 18 to 27 years old. At the time of the study, 138 of the participants were pursuing an undergraduate major in the university and 50 of them were workers. To measure the study variable, the

La presente investigación forma parte de los trabajos desarrollados por el cuerpo académico Educación y Sociedad del Centro Universitario de Los Altos de la Universidad de Guadalajara

2 Estudiante de Licenciatura en Psicología.

3 Doctor en Enseñanza de las Ciencias.

4 Doctor en Psicología. Dirección de correspondencia: Centro Universitario de Los Altos de la Universidad de Guadalajara. Carretera a Yahualica km 7.5 Cp. 44600. Teléfono 3787828033 Ext.: 56919. Tepatitlán de Morelos, Jalisco, México. Correo de correspondencia: ulises.reynoso@academicos.udg.mx

5 Licenciado en Psicología.

6 Estudiante de Licenciatura en Psicología.
} 
Resilience Scale was used in its translated and adapted version to Spanish. Results. The main finding is that university students obtain significantly higher scores than workers, both in the total resilience test $(t(186)=$ $-3.98, p=0.000, d=0.65)$ and in the two dimensions that compose it, Personal competence $(t(186)=-4.45, p$ $=0.000, d=0.73)$ and Acceptance of self and life $(t(186)=-2.23, p=0.026, d=0.36)$. Conclusion. The general conclusion indicates that a higher level of education could be considered a protective factor against adversity, as it is related to greater resilience.

Keywords. Resilience, students, young, resiliency.

\section{Resiliência. Diferencias entre estudantes universitários e jovens trabalhadores}

\section{Resumo}

Escopo. Comparar os níveis de resiliência entre jovens universitários e jovens trabalhadores, e identificar possíveis relações desta variável com a idade dos participantes. Metodologia. A amostra, do tipo incidental, esteve conformada por 188 jovens, com idade que oscilava entre os 18 e os 27 anos. Ao momento do estudo, 138 cursavam uma carreira profissional universitária e 50 eram trabalhadores. Para medir a variável do estudo foi utilizada a Escada de Resiliência traduzida e adaptada ao espanhol. Resultados. A principal descoberta é que os jovens universitários obtêm pontuações significativamente maiores que os trabalhadores, tanto no total da prova de resiliência $(t(186)=-3.98, p=0.000, d=0.65)$ como nas duas dimensões que a compõem, Competência pessoal $(t(186)=-4.45, p=0.000, d=0.73)$ e Aceitação de si mesmo e da vida $(t(186)=-2.23$, $p=0.026, d=0.36$ ). Conclusão. Um maior nível mais de estudos pode ser considerado um fator de proteção ante a adversidade, pois está relacionado à maior resiliência.

Palavras-chave. Resiliência, estudantes universitários, jovens, escada de resiliência.

\section{Introducción}

La investigación en torno a la resiliencia es un fenómeno relativamente nuevo, como señala Smith-Osborne (2007). Su estudio comenzó en los años 50; sin embargo, apenas en los años 80 se realizaron los primeros estudios sistemáticos. Pese a ello, dicho constructo cuenta ya con un amplio número de definiciones. Grotberg (1995) señala que la resiliencia corresponde a la capacidad del ser humano para hacer frente a las adversidades de la vida e, incluso, ser transformado por ellas. Por su parte, Wagnild y Young (1993) la consideran una característica de la personalidad que modera el efecto negativo del estrés y fomenta la adaptación, lo que conlleva fortaleza emocional.

Más recientemente, Windle, Bennett y Noyes (2011) apuntan que la resiliencia es un proceso dinámico en el que el individuo manifiesta habilidades adaptativas positivas, a pesar de haber sufrido traumas o adversidad. Según Masten y Coatsworth (1998), para hablar de resiliencia, es necesaria la presencia de una amenaza, riesgo o adversidad en la vida del individuo y que la calidad de la adaptación frente al riesgo o adversidad sea evaluada como buena.

Más allá de la distinción entre los términos utilizados por los autores que se han dado a la tarea de conceptualizarla, la mayoría concuerdan en considerar que la resiliencia es la capacidad humana de superar o sobrellevar de un modo favorable situaciones adversas o estresantes que pueden ocasionar consecuencias negativas (Caldera, Aceves y Reynoso, 2016; González-Arratia, ValdezMedina y Zavala-Borja, 2008; Polo, 2009). Además, la resiliencia implica un enriquecimiento de cualidades y factores de protección ante la situación adversa que se le presenta a la persona (González y Artuch, 2014).

Cabe aclarar que la resiliencia se distingue de la adaptación, en general, cuando se presenta en situaciones en las que la desadaptación sería lo esperado, pues, como señala Luthar (2006), 
"Ia resiliencia, por definición, abarca procesos atípicos, en el sentido de que la adaptación positiva se manifiesta en circunstancias de la vida que generalmente llevan a desajustes" (p. 739).

El estudio de la resiliencia se ha abordado desde dos enfoques: (a) desde la perspectiva del riesgo, la cual se centra en los aspectos que pueden desencadenar un daño biopsicosocial, es decir, se centra en los factores de riesgo alrededor del individuo y en cómo, a través de la resiliencia, son sobrellevados; y (b) desde la perspectiva del modelo de desafío, el cual hace hincapié en los factores protectores que favorecen en el individuo la capacidad o la habilidad para superar esas adversidades, es decir, la resiliencia (Polo, 2009).

A pesar del énfasis de las visiones en uno de los aspectos o factores (de riesgo o protectores), dichos enfoques no necesariamente se contraponen, por el contrario, su integración resulta complementaria, ya que, por un lado, se identifican las variables que pueden afectar negativamente $y$ de manera considerable al individuo, y por el otro, se contempla la existencia de barreras que pueden ayudar no solo a sobrellevar situaciones riesgosas, sino a sacarle el mayor provecho a la adversidad, lo cual conduce a una mejor calidad de vida para el individuo (Caldera et al., 2016).

Desde las dos perspectivas, los estudios se han dado a la tarea de identificar los factores o fenómenos que generan en los individuos mayores o menores capacidades resilientes, entre los que se incluyen tanto variables o dimensiones internas (por ejemplo, la inteligencia emocional y las habilidades de socialización) como factores externos (por ejemplo, la familia, el contexto social y las relaciones de amistad) (González-Arratia et al., 2008).

Uno de los ámbitos clásicos de los estudios sobre resiliencia es el contexto hospitalario o de la salud, tanto en pacientes con distintas patologías como en personas a cargo del cuidado de diversos tipos de pacientes, los cuales han diferenciado las habilidades resilientes según la patología de la que se habla (Crespo y Fernández, 2015; Dias et al., 2015; Vinaccia, Quiceno, Medina, Castañeda y Castelblanco, 2014).

Muchas de las investigaciones suelen centrarse en las distintas etapas del desarrollo evolutivo en población infantil, como las de Morelato (2014),
Quezada, González y Mecott (2014) y VeraBachmann y López (2014), las cuales han hecho énfasis en el estilo de crianza y su influencia en el desarrollo de habilidades resilientes. Existen también investigaciones en adultos mayores como las de Pimentel y Pinho (2015) y la de Portella y Liberalesso (2015), que destacan la satisfacción económica, la longitud de la jubilación y la planificación de esta como aspectos asociados a mayores niveles de resiliencia.

También hay un buen número de investigaciones que se han centrado en la juventud y la adolescencia, las cuales vinculan la resiliencia con el consumo de drogas, el bienestar psicológico subjetivo, la autoestima y algunos aspectos sociodemográficos como el nivel socioeconómico y educativo (Álvarez, Alonso y Guidorizzi 2014; González y Oliva, 2017; Gutiérrez y Romero 2014; Morales y González, 2014; Quiceno, Mateus, Cárdenas, Villareal y Vinaccia, 2013). Cabe decir que los estudios sobre resiliencia se han extendido a distintas poblaciones y contextos regionales (Forján y Morelatto, 2018; Sart, Börkan, Erkman y Serbest, 2016; Villalta, Delgado, Escurra y Torres, 2017).

Específicamente centrados en jóvenes y el contexto educativo universitario, destaca el estudio llevado a cabo por Limonero, Tomás, Fernández, Gómez y Ardilla (2012), con alumnos universitarios en España, en el que se encontró que el 20.1\% podían ser considerados resilientes y que la resiliencia es un factor que afecta significativamente la satisfacción con la vida. Otro estudio español desarrollado por Ríos, Carrillo y Sabuco (2012) en una muestra de alumnos de Enfermería, reportó que el $14.7 \%$ de la población obtuvo bajos niveles de resiliencia. González y Artuch (2014), también en España, realizaron una investigación que permitió denotar altos niveles de resiliencia en estudiantes universitarios. Además, se encontró una relación entre el sexo y el tipo de universidad (siendo las mujeres de instituciones educativas religiosas quienes obtuvieron puntajes mayores). En Australia, la investigación de Schofield, O'Halloran, McLean y Forrester-Knauss (2016) permitió identificar la resiliencia como un factor contra la conducta depresiva en estudiantes universitarios.

En cuanto a estudios realizados en Latinoamérica, resultan de interés los hallazgos de Rivas (2012), quien en una investigación con estudiantes de la carrera de Medicina de una 
universidad venezolana, reportó altos niveles de resiliencia. En su trabajo, caracterizó la resiliencia como una aceptación positiva al cambio, competencias para asumirlo, autocontrol y una espiritualidad significativa. Este estudio reveló relaciones importantes entre la resiliencia y la edad de los estudiantes, siendo los más jóvenes quienes mostraron más capacidades resilientes. Con resultados similares, el estudio de Álvarez y Cáceres (2010) demostró una relación significativa entre la resiliencia y la edad, el sexo (siendo las mujeres las que mostraron mejores niveles de resiliencia), el nivel socioeconómico y la cantidad de hijos.

En México existen algunas investigaciones en jóvenes estudiantes, por ejemplo, un estudio realizado por Velasco, Suárez, Córdova, Luna y Mireles (2015) con estudiantes de Enfermería, cuyo objetivo era estimar el grado de resiliencia, permitió establecer que el $63 \%$ de los participantes tenían un nivel alto de resiliencia. Asimismo, se hizo una asociación de estos puntajes con altos niveles de rendimiento académico, no haber repetido asignaturas o tener exámenes extraordinarios, así como tener una familia funcional.

Por su parte, González (2011) evaluó distintos factores resilientes en estudiantes de primer ingreso en la carrera de Medicina en la Ciudad de México. En este estudio se destacaron la interacción social, la introspección y la moralidad como las dimensiones de la resiliencia con puntajes más altos, mientras que la independencia, la creatividad, el humor y el pensamiento crítico fueron los factores de la resiliencia con puntuaciones más bajas. También se presentaron diferencias significativas entre hombres y mujeres. Otro estudio realizado con población universitaria del estado de Jalisco encontró altos niveles de resiliencia en estudiantes de las carreras de Derecho, Negocios Internacionales, Medicina Humana y Psicología; además, reportó que no existen diferencias entre ellas, así como tampoco entre el sexo de los participantes (Caldera et al., 2016).

Referido lo anterior y reconociendo la importancia que en la actualidad tiene el estudio de la resiliencia, el presente trabajo, desarrollado bajo el enfoque cuantitativo con un diseño no experimental y transversal y de alcance comparativo (Hernández, Fernández y Baptista, 2010), se plantearon los siguientes objetivos: (a) comparar los niveles de resiliencia entre jóvenes universitarios y jóvenes trabajadores, (b) comparar dichos niveles entre hombres y mujeres, e (c) identificar posibles relaciones de dicha variable con la edad de los participantes. Las hipótesis que guiaron la investigación fueron: (a) existen diferencias en los niveles de resiliencia entre jóvenes universitarios y jóvenes trabajadores, (b) son distintos los niveles de resiliencia entre los hombres y las mujeres, y (c) la relación entre los niveles de resiliencia y la edad de los participantes es significativa.

\section{Método}

\section{Participantes}

La muestra fue de tipo incidental y estuvo integrada por 188 jóvenes, cuya edad oscilaba entre los 18 y los 27 años $(M=20.66, D E=1.861)$. En el momento en que se efectuó el trabajo de campo, 138 estaban cursando una carrera profesional universitaria de una institución de educación superior pública $(73.4 \%) \quad\left(M_{\text {edad }}=20.51, D E=2.023\right)$, y $50(26.6 \%)$ $\left(M_{\text {edad }}=21.10, D E=1.861\right)$ eran jóvenes que laboraban y contaban con el bachillerato (nivel medio superior) como máximo grado de estudios. La pesquisa se llevó a cabo en el entorno regional conocido como los Altos de Jalisco, México. Participaron 97 mujeres $(51.6 \%) \quad\left(M_{\text {edad }}=20.70\right.$, $D E=1.953)$ y 91 hombres $(48.4 \%)\left(M_{\text {edad }}^{\text {edad }}=20.63\right.$, $D E=1.768)$. Los criterios de inclusión fueron ser jóvenes entre los 18 y los 30 años, y que residieran en el entorno regional comentado. Los de exclusión consistieron en que los jóvenes universitarios no laboraran, y los trabajadores no cursaran o hubiesen cursado estudios universitarios.

\section{Instrumento}

Se utilizó la Escala de Resiliencia de Wagnild y Young (1993), específicamente la versión traducida y adaptada al español por Heilemann, Lee y Kury (2003). La validez de constructo se corroboró, en los dos trabajos citados, a través de análisis factoriales que arrojaron dos dimensiones o factores: competencia personal ("cuando estoy en una situación difícil generalmente encuentro la salida") y aceptación de sí mismo y de la vida ("mi vida tiene significado"). Los índices de consistencia interna reportados fueron 0.89 y 0.93 , respectivamente. El Alfa de Cronbach de este estudio fue $\alpha=0.88$. En total, la prueba estuvo integrada por un total de 25 
reactivos, en escala tipo Likert con siete opciones de respuesta (desde 1 [totalmente en desacuerdo] hasta 7 [totalmente de acuerdo]). También se identificaron varios niveles: bajo (25 a 120), medio (121 a 146) y alto (147 a 175). A mayor puntuación mayor resiliencia (Caldera et al., 2016).

\section{Procedimiento}

Para desarrollar la presente investigación, se contó con la anuencia del Comité de Bioética de una institución de educación superior pública del estado de Jalisco, México. Con la intención de suministrar la Escala de Resiliencia, en el caso de los universitarios, se acudió a sus respectivos salones de clase; y para el caso de los jóvenes trabajadores, se asistió a los lugares en donde laboraban. La aplicación del instrumento la realizaron directamente los responsables de la investigación. En el caso de los estudiantes de nivel superior, la recolección de la información fue grupal y tuvo una duración aproximada de 15 minutos por salón de clases; mientras que con los trabajadores fue individual, con una duración promedio de 20 minutos. El trabajo de campo se desarrolló en el segundo trimestre de 2018.

Respecto a los aspectos éticos, antes de comenzar la recolección de los datos se explicó a los participantes el objetivo central del estudio y se aseguró el anonimato y confidencialidad de las respuestas; además, quienes no estuvieran dispuestos a colaborar en la investigación, estaban en su derecho de no participar. No se utilizó el consentimiento informado, ya que, de acuerdo con la norma oficial mexicana sobre investigación NOM-012-SSA3-2012, en los casos de estudios sin riesgo o con riesgo mínimo que emplean pruebas psicológicas a individuos o grupos en los que no se manipula la conducta del sujeto, no es necesario emplear dicho requerimiento ético (Secretaría de Gobernación, 2012).

\section{Análisis de los datos}

En primer término, se obtuvieron medias, desviaciones estándar y frecuencias de las variables de estudio. Mediante la prueba Kolmogorov, se corroboró la normalidad de la variable de estudio $(z=1.09, p=0.186)$. Asimismo, para identificar el criterio de homocedasticidad, se empleó la prueba de Levene, obteniéndose homogeneidad de dicha variable entre los grupos de universitarios y no universitarios $(p=0.121)$ y ausencia de la misma entre sexos $(p=0.038)$. Por ello, para la comparación de la resiliencia entre los primeros dos grupos, se empleó la $t$ de student para muestras independientes. El tamaño del efecto se calculó mediante el índice $d$ de Cohen; y para el caso de las diferencias respecto del sexo, se utilizó la prueba $U$ de Mann-Whitney. La dimensión de las diferencias se obtuvo con apoyo de la $r$ de Rosenthal. Finalmente, para identificar correlaciones entre la edad y la resiliencia, se ejecutó un procedimiento de correlación a través del coeficiente Rho de Spearman. Dichos análisis se llevaron a cabo mediante el paquete estadístico SPSS 25.

\section{Resultados}

Conforme a los parámetros expresados anteriormente, los resultados revelaron un puntaje medio en el total de la prueba de resiliencia $(M=141.68, D E=16.52)$. En el caso de la dimensión competencia personal, se obtuvo una media de $97.46(D E=11.74)$, y en la de aceptación de sí mismo y de la vida, de $44.16(D E=6.17)$.

Como se muestra en la tabla 1 , respecto de las frecuencias en cada uno de los niveles de resiliencia, se advierte que $14(7.4 \%)$ de las 188 personas abordadas en el estudio presentaron niveles bajos y $77(41 \%)$, altos.

Tabla 1

Niveles de resiliencia

\begin{tabular}{ccc}
\hline Nivel de resiliencia & Frecuencia & Porcentaje \\
\hline Bajo & 14 & 7.4 \\
Medio & 97 & 51.6 \\
Alto & 77 & 41.0 \\
Total & 188 & 100.0 \\
\hline
\end{tabular}

Fuente: elaboración propia.

Para la comparación de los niveles de resiliencia entre universitarios y trabajadores, se optó por la prueba paramétrica $t$ de student, en la que se advirtieron diferencias significativas tanto en el total como en los factores que conforman la escala, siendo, en todos los casos, superiores las medias de quienes estudian carreras profesionales 
(tabla 2). Además, el tamaño del efecto (calculado mediante el índice $d$ de Cohen) fue mediano en el total de resiliencia y en el factor competencia personal, y pequeño en la dimensión aceptación de sí mismo y de la vida.

Tabla 2

Comparación de los niveles de resiliencia entre jóvenes universitarios y jóvenes trabajadores

\begin{tabular}{lccccccccc}
\hline \multirow{2}{*}{ Variable/factor } & \multicolumn{3}{c}{ Universitarios } & \multicolumn{2}{c}{ No universitarios } & & & \\
\cline { 2 - 6 } & $M$ & $D E$ & $M$ & $D E$ & & & $p$ & $d$ \\
\hline Resiliencia (total) & 144.46 & 14.10 & 134.00 & 20.23 & -3.98 & 0.000 & 0.65 \\
Competencia personal & 99.64 & 9.92 & 91.44 & 14.02 & -4.45 & 0.000 & 0.73 \\
Aceptación de sí mismo y de la vida & 44.82 & 5.48 & 42.56 & 7.57 & -2.23 & 0.026 & 0.36 \\
\hline
\end{tabular}

Fuente: elaboración propia.

Para identificar diferencias en los niveles de resiliencia según el sexo de los participantes, se optó por la versión no paramétrica de la $t$ de student, es decir, la prueba $U$ de Mann-Whitney. Como se puede apreciar en tabla 3, las diferencias significativas se encuentran en el factor competencia personal, y se presenta un mayor nivel en el caso de los hombres. Para dimensionar el tamaño del efecto de tal diferencia, se utilizó el índice $r$ de Rosenthal $(r=0.530)$. De acuerdo con Grissom y Kim (2005), el tamaño de tal efecto podría clasificarse como pequeño.

Tabla 3

Comparación de los niveles de resiliencia entre hombres y mujeres

\begin{tabular}{lcccccccc}
\hline \multirow{2}{*}{ Variable/factor } & \multicolumn{2}{c}{ Mujeres } & \multicolumn{2}{c}{ Hombres } & & & $R$ \\
\cline { 2 - 6 } & $M$ & $D E$ & $M$ & $D E$ & & & $p$ & $R$ \\
\hline Resiliencia (total) & 139.37 & 18.08 & 144.14 & 13.35 & -1.52 & 0.127 & 0.11 \\
Competencia personal & 95.63 & 12.93 & 99.42 & 9.954 & -2.03 & 0.042 & 0.14 \\
Aceptación de sí mismo y de la vida & 43.74 & 7.03 & 44.73 & 5.104 & -0.67 & 0.498 & 0.04 \\
\hline
\end{tabular}

Fuente: elaboración propia.

Por último, se analizó la posible correlación entre la variable de edad y el nivel de resiliencia de los participantes. Tomando en cuenta la falta de normalidad de la variable edad, se utilizó la prueba
Rho de Spearman (tabla 4). Se encontró una relación significativa entre la edad y el factor aceptación de sí mismo y de la vida; sin embargo, la fuerza de la relación fue débil ( $r$ ho $=0.17$ ).

Tabla 4

Correlación entre la edad de los jóvenes y las puntuaciones de resiliencia

\begin{tabular}{clll}
\hline Variables & $\begin{array}{c}\text { Resiliencia } \\
\text { (total) }\end{array}$ & $\begin{array}{c}\text { Competencia } \\
\text { personal }\end{array}$ & $\begin{array}{c}\text { Aceptación de sí } \\
\text { mismo y de la vida }\end{array}$ \\
\hline \multirow{2}{*}{ Edad Rho } & 0.13 & 0.090 & $0.17^{*}$ \\
\cline { 2 - 4 }$p$ & 0.089 & 0.218 & 0.017 \\
\hline
\end{tabular}

Fuente: elaboración propia. 


\section{Discusión}

Las puntuaciones de resiliencia de la población estudiada, de acuerdo con los resultados, fueron en promedio de $141.68(D E=16.52)$, lo cual corresponde al nivel medio, según la prueba. Dichos resultados difieren con los obtenidos tanto en los distintos contextos y con población universitaria como en la ciudad de México (Velasco et al., 2015), en Venezuela (Rivas, 2012) y en España (Ríos et al., 2012), en cuyos casos la resiliencia reportó índices superiores.

Conforme a la primera hipótesis, se encontró que los estudiantes universitarios obtienen mayores puntuaciones $(M=144.46)$ de resiliencia en comparación con quienes se identificaron como trabajadores $(M=134.00)$. Estos resultados concuerdan con los encontrados por González y Artuch (2014), Ríos et al. (2012) y Velasco et al. (2015), quienes advirtieron que los estudiantes universitarios obtienen en su mayoría puntajes altos de resiliencia.

Esta circunstancia puede deberse a la propia exigencia de la educación superior, pues los jóvenes que acceden a dicho nivel debieron transitar de manera obligatoria por al menos 12 años de estudios previos, en los cuales se enfrentaron a muchas circunstancias complejas y momentos problemáticos (como el estrés, las evaluaciones, los conflictos grupales y las demandas de los profesores y padres de familia, entre otras) que exigen la adquisición de conductas y cogniciones con un cierto grado de resiliencia (Caldera et al., 2016). Sin embargo, dicha aseveración requiere de mayor indagación empírica, sobre todo porque no existen elementos suficientes para estimar que los jóvenes trabajadores no enfrentan situaciones adversas ante las cuales tengan que mostrar conductas resilientes.

Por tales razones, resulta pertinente realizar más estudios al respecto, en los que se tenga mayor delimitación y control de los factores sociodemográficos de los participantes (por ejemplo: tipo de trabajo, tiempo de permanencia, nivel máximo de estudios alcanzado por los jóvenes trabajadores o la carrera en la que participan los jóvenes universitarios) y se amplíe significativamente el tamaño de las muestras.

Con relación a la segunda hipótesis, se observó una tendencia por parte de los hombres a obtener un puntaje más alto en el factor de competencia personal. Esta situación es contraria a estudios anteriores, los cuales reportan que las mujeres obtienen puntajes más altos (Álvarez y Cáceres, 2010; González, 2011; GonzálezArratia y Valdez-Medina, 2013), y a otros que no advirtieron diferencias (Walter-Ginzburg, Shmotkin, Blumstein y Shorek, 2005). A manera de conjetura (que en su momento debiera probarse), dichos resultados pudieran deberse a que, en el contexto del estudio, a los integrantes del sexo masculino se les presenta un entorno más competitivo en materia de consecución de logros, además, de manera continua, son evaluados a nivel social y familiar.

Respecto de la tercera hipótesis, no se encontraron relaciones significativas entre la edad y el total de la prueba de resiliencia y el factor competencia personal. Esta condición difiere de los hallazgos en el estudio con estudiantes de Medicina venezolanos (Rivas, 2012), ya que este investigador encontró correlaciones significativas y negativas entre ambas variables. Únicamente se advirtieron relaciones significativas (en este caso, positivas) entre la edad y el factor de aceptación de sí mismo y de la vida. Este hecho puede argumentarse al sostener que la resiliencia es una capacidad que se desarrolla a través de procesos dinámicos durante el ciclo de vida y, en especial, porque los factores protectores internos son dominios adquiridos con la experiencia y por la necesidad de resolución de problemas (Plata, citado en González-Arratia \& Valdez-Medina, 2013). Pero tal apreciación debe interpretarse con cautela, debido a que la fuerza de correlación encontrada fue baja.

En cuanto a las limitaciones del presente estudio, se pueden señalar las siguientes: la muestra empleada fue relativamente pequeña y el control de variables fue limitado, ya que la cantidad de personas en cada grupo no fue suficientemente equitativa, delimitada o caracterizada para proporcionar un sólido sustento a las conclusiones y a la condición de haber utilizado un instrumento de recolección de datos tipificado como autoinforme, es decir, que los niveles de resiliencia se obtienen mediante ejercicios de percepción de los participantes.

Por tales razones y en términos de prospectiva, se sugiere desarrollar estudios longitudinales que permitan explicar mejor los niveles de resiliencia en la población de estudiantes universitarios de los Altos de Jalisco. También, y dado que uno de los objetivos centrales de la investigación fue comparar 
los niveles de resiliencia entre jóvenes universitarios y jóvenes trabajadores, se propone ampliar la población estudiada e incluir en el análisis una mayor cantidad de aspectos sociodemográficos o escolares (como el nivel socioeconómico de los jóvenes, el tipo de trabajo, la permanencia, el nivel de estudios alcanzado o el tipo de carrera que cursan). Además, se considera necesario continuar con investigaciones que permitan identificar otros factores propios del proceso educativo y del paso por la universidad asociados a la resiliencia, para que la relación resiliencia-estudios superiores pueda ser caracterizada de manera más precisa.

Como conclusión general, se puede señalar que contar con un mayor nivel de estudios (en este caso, de nivel superior universitario), podría considerarse un factor protector ante la adversidad; por consiguiente, sería deseable que tanto a nivel personal, familiar como institucional se realizaran mayores esfuerzos para aumentar el nivel educativo de las personas. Adicionalmente, se debe desarrollar un mayor número investigaciones de corte explicativo y sobre todo aplicado, cuyo propósito central sea la mejora en el nivel de resiliencia de la población.

\section{Referencias}

Álvarez, A., Alonso, M. y Guidorizzi, A. (2014). Factores predictivos del uso de alcohol y tabaco en los adolescentes. Revista LatinoAmericana de Enfermagem, 22(6), 1056-1062. doi:10.1590/0104-1169.3570.2516

Álvarez, L. y Cáceres, L. (2010). Resiliencia, rendimiento académico y variables sociodemográficas en estudiantes universitarios de Bucaramanga. Psicología Iberoamericana, 18(2), 37-46. Recuperado de https://www. redalyc.org/articulo.oa?id=133915921005

Caldera, J., Aceves, B. y Reynoso, Ó. (2016). Resiliencia en estudiantes universitarios. Un estudio comparado entre carreras. Psicogente, 19(36), 227-239. doi:10.17081/ psico.19.36.1294

Crespo, M. y Fernández, V. (2015). Resiliencia en cuidadores familiares de personas mayores dependientes. Anales de Psicología, 31(1), 1927. doi: 10.6018/analesps.31.1.158241
Dias, R., Santos, R., Barroso, M., Lima, M., Torres, B., ... Nascimento, M. (2015). Resilience of Caregivers of People with Dementia: A Systematic Review of Biological and Psychosocial Determinants. Trends in Psychiatry and Psychotherapy, 37(1), 12-19. http://dx.doi.org/10.1590/2237-6089-20140032

Forján, R. y Morelatto, G. (2018). Estudio comparativo de factores de resiliencia en docentes de contextos socialmente vulnerables. Psicogente, 21(40), 277-296. doi:10.17081/ psico.21.40.3075

González, M. A. (2011). La resiliencia y el engagement y su asociación con el rendimiento académico en los estudiantes de la Licenciatura de médico cirujano de la Facultad de Medicina de la Universidad Autónoma del Estado de México (Tesis para obtener la especialidad en Salud Pública). Universidad Autónoma del Estado de México, México.

González, M. y Artuch, R. (2014). Perfiles de resiliencia y estrategias de afrontamiento en la universidad: Variables contextuales y demográficas. Electronic Journal of Research in Educational Psychology, 12(3), 621-648. doi:10.14204/ejrep.34.14032

González, S. y Oliva, E. (2017). Análisis de la vulnerabilidad y resiliencia económica de Baja California en el contexto de la crisis financiera internacional. Frontera Norte, 29(58), 141-169. https://dx.doi.org/10.17428/rfn.v29i58.536

González-Arratia, N. y Valdez-Medina, J. L. (2013). Resiliencia: diferencias por edad en hombres y mujeres mexicanos. Acta de Investigación Psicológica, 3(1), 941-955. Recuperado de http://www.scielo.org.mx/ scielo.php?script=sci_arttext\&pid=S200748322013000100004\&lng=es\&tlng=es

González-Arratia, N., Valdez-Medina, J. L. y ZavalaBorja, Y. (2008). Resiliencia en adolescentes mexicanos. Enseñanza e Investigación en Psicología, 13(1), 41-52. Recuperado de https:// www.redalyc.org/pdf/292/29213104.pdf

Grissom, R., \& Kim, J. (2005). Effect Sizes for Research. A Broad Practical Approach. Mahwah: LEA. 
Grotberg, E. (1995). The International Resilience Project: Promoting Resilience in Children. Wisconsin: University of Wisconsin.

Gutiérrez, M. y Romero, I. (2014). Resiliencia, bienestar subjetivo y actitudes de los adolescentes hacia el consumo de drogas en Angola. Anales de Psicología, 30(2), 608-619. doi:10.6018/analesps.30.2.148131

Heilemann, M., Lee, K., \& Kury, F. (2003). Psychometric Properties of the Spanish Version of the Resilience Scale. Journal of Nursing Measurement, 11(1) 61-72. doi: 10.1891/ jnum.11.1.61.52067

Hernández, R., Fernández, C. y Baptista M. (2010). Metodología de la investigación. México: McGraw-Hill.

Limonero, J., Tomás, J., Fernández, J., Gómez, M. y Ardilla, A. (2012). Estrategias de afrontamiento resilientes y regulación emocional: predictores de satisfacción con la vida. Behavioral Psychology, 20(1), 183-196. Recuperado de https://dialnet.unirioja.es/servlet/ articulo?codigo $=3924266$

Luthar, S. (2006). Resilience in Development: A Synthesis of Research across Five Decades. In D. Cicchetti, \& D. J. Cohen (Eds.), Developmental Psychopathology: Risk, Disorder, and Adaptation (pp. 739-795). Hoboken: John Wiley \& Sons.

Masten, A., \& Coatsworth, J. (1998). The Development of Competence in Favorable and Unfavorable Environments: Lessons from Research on Successful Children. American Psychologist, 53(2), 205-220. doi: 10.1037/0003-066X.53.2.205

Morales, M. y González, A. (2014). ResilienciaAutoestima-Bienestar psicológico y Capacidad intelectual de estudiantes de cuarto medio de buen rendimiento de liceos vulnerables. Estudios Pedagógicos, 40(1), 215-228. doi:10.4067/S0718-07052014000100013

Morelato, G. (2014). Evaluación de factores de resiliencia en niños argentinos en condiciones de vulnerabilidad familiar. Universitas Psychologica, 13(4), 1473-1488. doi:10.11144/ Javeriana.UPSY13-4.efrn
Pimentel, C., \& Pinho, L. (2015). The Importance of Resilience for Well-Being in Retirement. Paidéia, 25(61), 191-199. doi:10.1590/198243272561201507

Polo, C. (2009). Resiliencia: Factores protectores en adolescentes de 14 a 16 años (Tesis para obtener el grado de Licenciado en Psicología). Universidad del Aconcagua, Argentina. Recuperado de http://bibliotecadigital.uda.edu. ar/objetos_digitales/71/tesis-1426-resiliencia. pdf

Portella, A. y Liberalesso, A. (2015). Resilience in Aging: Literature Review. Ciência y Saúde Coletiva, 20(5), 1475-1495. doi:10.1590/141381232015205.00502014

Quezada, L., González, T. y Mecott, G. (2014). Resiliencia en pacientes pediátricos sobrevivientes de quemaduras. Acta de Investigación Psicológica, 4(2), 1585-1594. Recuperado de http://www.scielo.org.mx/ scielo.php?script=sci_arttext\&pid=S200748322014000200010\&lng=es\&tlng=es

Quiceno, J., Mateus, J., Cárdenas, M., Villareal, D. y Vinaccia, S. (2013). Calidad de vida, resiliencia e ideación suicida en adolescentes víctimas de abuso sexual. Revista de Psicopatología y Psicología Clínica, 18(2), 107-117. Recuperado de http://revistas.uned.es/index.php/RPPC/ article/viewFile/12767/pdf_3

Ríos, M., Carrillo, C. y Sabuco, E. (2012). Resiliencia y Síndrome de Burnout en estudiantes de enfermería y su relación con variables sociodemográficas y de relación interpersonal. International Journal of Psychological Research, 5(1), 88-95. Recuperado de https://dialnet. unirioja.es/servlet/articulo?codigo=5134686

Rivas, S. (2012). Fortalezas de carácter y resiliencia en estudiantes de Medicina de la Universidad de los Andes, Mérida, Venezuela (Tesis para obtener el grado de Doctor en Patología Existencial e Intervención en Crisis). Universidad Autónoma de Madrid, Madrid.

Sart, Z. H., Börkan, B., Erkman, F., \& Serbest, S. (2016). Resilience as a Mediator between Parental Acceptance-Rejection and Depressive Symptoms among University Students in Turkey. Journal of Counseling and Development, 94(2), 195-209. doi:10.1002/jcad.12076 
Schofield, M., O'Halloran, P., McLean, S., \& ForresterKnauss, C. (2016). Depressive Symptoms among Australian University Students: Who is at Risk? School of Psychology and Public Health, 51(2), 135-144. doi:10.1111/ap.12129

Secretaría de Gobernación. (2012). NOM-012SSA3-2012. Que establece los criterios para la ejecución de proyectos de investigación para la salud en seres humanos. Diario Oficial de la Federación. Estados Unidos Mexicanos, 4 de enero de 2013. Recuperado de http://dof. gob.mx/nota_detalle.php?codigo $=5284148 \&$ fe cha $=04 / 01 / 2013$

Smith-Osborne, A. (2007). Life Span and Resiliency Theory: A Critical Review. Advances in Social Work, 8(1), 152-168. doi: https://doi. org/10.18060/138

Velasco, V., Suárez, G., Córdova, S., Luna, L. y Mireles, S. (2015). Niveles de resiliencia en una población de estudiantes de licenciatura y su asociación con variables familiares y académicas. Revista Iberoamericana de Producción Académica y Gestión Educativa, 2(3) 1-23. Recuperado de https://www.pag.org. $\mathrm{mx} /$ index.php/PAG/article/view/490

Vera-Bachmann, D. y López, M. (2014). Resiliencia académica: una alternativa a explorar en la educación de niños y niñas en contextos rurales. Integra Educativa, 7(2), 187-205. Recuperado de http://www.scielo.org.bo/ scielo.php?script=sci_arttext\&pid=S199740432014000200012\&lng=es\&tlng=es
Villalta, M., Delgado, A., Escurra, L. y Torres, W. (2017). Resiliencia y rendimiento escolar en adolescentes de Lima y de Santiago de Chile de sectores vulnerables. Universitas Psychologica, 16(4), 1-10. doi:10.11144/Javeriana.upsy16-4. rrea

Vinaccia, S., Quiceno, J., Medina, A., Castañeda, B. y Castelblanco, P. (2014). Calidad de vida, resiliencia, percepción y conducta de enfermedad en pacientes con diagnóstico de dispepsia funcional. Acta Colombiana de Psicología, 17(1), 61-70. doi:10.14718/ ACP.2014.17.1.7

Wagnild, G., \& Young, H. (1993). Development and Psychometric Evaluation of the Resilience Scale. Journal of Nursing Measurement, 1(2), 165-178. Recuperado de https://www.ncbi. nlm.nih.gov/pubmed/7850498

Walter-Ginzburg, A., Shmotkin A., Blumstein, B., \& Shorek, A. (2005). A Gender-Based Dynamic Multidimensional Longitudinal Analysis of Resilience and Mortality in the Old-Old in Israel: The Cross-Sectional and Longitudinal Aging Study. Social Science \& Medicine, 60(8), 17051715. doi: 10.1016 / j.socscimed.2004.08.023

Windle, G., Bennett, K., \& Noyes, J. (2011). A Methodological Review of Resilience Measurement Scales. Health and Quality of Life Outcomes, 9, 1-18. doi: 10.1186 / 14777525-9-8

Para citar este artículo / To cite this article / Para citar este artigo: Hernández-León, J. A., Caldera-Montes, J. F., Reynoso-González, O. U., Caldera-Zamora, I. A. y Salcedo-Orozco, S. (2020). Resiliencia. Diferencias entre estudiantes universitarios y jóvenes trabajadores. Pensamiento Psicológico, 18(1), 21-30. doi:10.11144/Javerianacali.PPSI18-1.rdeu 